\title{
A Robust Digital Watermarking Scheme Based on Bandelet and Image Fusion
}

\author{
Yuancheng Li and Jie Ma \\ School of Control and Computer Engineering, \\ North China Electric Power University, Beijing, China \\ ycli@ncepu.edu.cn,majie2012@foxmail.com
}

\begin{abstract}
In this paper, a robust digital watermarking scheme is proposed, in which the watermark is embedded several times in different places to improve robustness. First, an oxiginal image is transformed into a coefficients matrix through bandeletization. Then the watermark image is embedded into different places of the bandeletization coefficients matrix simultaneously guided by human visual system (HVS). Because the number of non-zero wavelet coefficients have been greatly decreased by bandletization and the most information has been concentrated on some regions, this scheme possessed a stronger robustness against the noise attacks. At last, the watermarked image is reconstructed through inverse bandelet transformation. In the process of extracting watermark image, the watermark image is extracted by using independent component analysis (ICA) and principle component analysis (PCA) and image fusion. Experimental results demonstrate that the proposed scheme is possess of a good performance in both imperceptibility and robustness.
\end{abstract}

Keywords: robust watermark, bandelet; image fusion

\section{Introduction}

Digital watermarking is the process of embedding or hiding digital information called watermark into a nuttimedia product, in which the embedded data can be extracted or detected from the watermarked product later for protecting digital content copyright and ensuring tamper-resistance, but is indiscernible and hard to remove by unauthorized persons [1].

According to the processing domain of the host multimedia product, digital image watermarking techniques can be roughly categorized as spatial-domain and frequency-domain methods [2]. Spatial domain watermarking is attractive because it provides a better intuition on how to attain an optimal tradeoff among robustness, capacity and imperceptibility. Thus, putting forward public spatial domain algorithms which survive a broad range of manipulations is an important issue. However, the most serious problem of spatia domain watermarking is the weakness of robustness. In frequency domain, the vatermark is inserted into transformed coefficients matrix of the host image. Therefore frequency domain watermarking methods possess stronger robustness against watermarking attacks and more hiding capacity as watermark information is spread out to entire host image[3]. Since frequency domain watermarking methods possess a good performance of robustness in comparison to spatial watermarking, it has become researchers' focus for image watermarking. Frequency domain concentrates the whole image into some spectral coefficients where watermarks can be embedded, just like DCT, DWT and RT (ridgelet transform) [4]. DWT based image watermarking methods are presented by Chuntao Wang et al. [5], N. Bi et al. [6], D. Kundur et al. [7], Mohsen Zareian et al. [8] and V. Sentthil et $a l$. [9]. It is widely accepted that the frequency domain methods can easily exploit the perceptual models based on characteristics of the human visual system (HVS) to match the best tradeoff between invisibility and robustness [10]. 
This paper proposes a robust digital watermarking scheme based on bandelet domain. The watermark image is embedded into the specific district of bandelet coefficients matrix, with the guidance of HVS, in order to enhance robustness and expand capacity.

The rest of this paper is organized as follows: Section 2 separately describes the watermark embedding and watermark extraction of the proposed watermarking method. In Section 3, the experimental results are presented to show the performance of the proposed watermark. Finally, we draw out the conclusions of this paper in Section 4.

\section{The Proposed Robust Watermarking Scheme}

Bandelet transform was first proposed by Mallat and Pennec [11], which is a multiscale geometric analysis arithmetic to overcome the weakness of wavelet in high-dimensional data. It defines a kind of geometric flow of vectors, which can represent geometrical regularity of the local image. This transform takes full use of geometrical regularity of the original image, but also has two significant weakness. First, because basis function of the bandelet transform is not globally orthogonal, the edge effect of the constructed image will appear. Second, there is too much computation. So, the second generatio bandelet was introduced by Mallat and Pennec [12]. In the bandelet transform, bandeletization is an important procedure by removing the redundancy of a warped wavelel transform. The second generation bandelet transform is constructed aren a standard orthogonal wavelet transform.

Traditional wavelet decomposition is pyramid-shaped. Images are decomposed into four equal parts, low frequency information, horizontal information, vertical information and diagonal information with orthogonal wavelet. And then low frequency information should continue to be decomposed into four equal parts as mentioned earlier until some conditions are satisfied.

Tree-shaped bandelet decomposition aecompose inages into an incomplete tree. This kind of decomposition not only conninue to decompose the low information, but also other parts, judged by the human visual characteristic. The feature of this kind of decomposition is very useful to hide a lot of information in inages.

According to human visulal system model, sensitivity of human's eyes show some features as follow: 1) rnore sensitive to variations of lightness in smooth area than texture area, 2) more sensitive to additional noise in low light area than high light area, 3) more sensitive to variations in marginal area than central area.[13]

In the bandeletization coefficients tree, low frequency area gathers lots of energy, which is suitable for embedding watermark, but it usually also is smooth area, which will contribute to visual sensitivity. On the contrary, high frequency area also is texture area, which is suitable for embedding watermark, but it gathers little energy, which will limit strength of the watermark. So, in the process of bandelet decomposition, reasonably applying one principle to judge whether this part should be decomposed and whether suitable for embedding watermark image is very important to achieve better balance between aforementioned two situations.

According to human's visual characteristic, frequency contrast sensibility and pixels' significance are separately computed to judge whether this part is suitable to be decomposed further. Frequency contrast sensitivity is computed by [14]:

$$
\mathrm{C}(\mathrm{u}, \mathrm{v})=5.05 e^{-0.178(u+v)}\left(e^{0.1(u+v)}-1\right)
$$

In this equation, $\mathrm{u}$ and $\mathrm{v}$ separately represent corresponding spatial frequency. By taking use of frequency contrast sensitivity matrix, visual sensitivity of an image block can be calculated for embedding watermarks and then decomposition principle of sub-tree gets clear. Specifically, it can be computed as [14]:

$$
\mathrm{S}=\sum_{\mathbb{W}(u, v)} C(u, v)|F(u, v)|^{2}
$$

$F(u, v)$ is Fourier transform of an image block $f(u, v)$ and s reflects visual sensitivity of human eyes to this image block. The larger the value of $s$ is, the less sensitive human eyes are to this image block. Therefore watermarks with higher strength can be embedded 
into this block. Meanwhile, this block could continue to be decomposed. In this paper, Eq. (2) is expanded to bandelet domain.

$$
S_{b}=\sum_{\forall(u, v)} C(u, v)\left|F_{b}(u, v)\right|^{2}
$$

$F_{b}(u, v)$ is bandelet transform of image block $f(u, v)$. Then, a threshold value $T_{e}$ can be computed by:

$$
T_{e}=\alpha \ln S_{b}
$$

$\alpha$ is the strength coefficient of embedding watermark process, obtained by experiment. An image block should continue to be decomposed to four equal parts as mentioned earlier until its $S_{b}$ is lower than $T_{e}$.

\subsection{The Embedding Algorithm}

First, 2D wavelet transform is applied to the host image, and then the host image is decomposed into four equal parts separately representing different frequency domain. Second, the bandeletization performs on all four parts in order to gain the bandeletization coefficient of the bandelet tree. Thirdly, watermarks are separately mbedded into corresponding suitable image blocks. At last, the watermarked image is reconstructed by taking inverse transform.

The steps of watermark embedding are shown in Figure 1

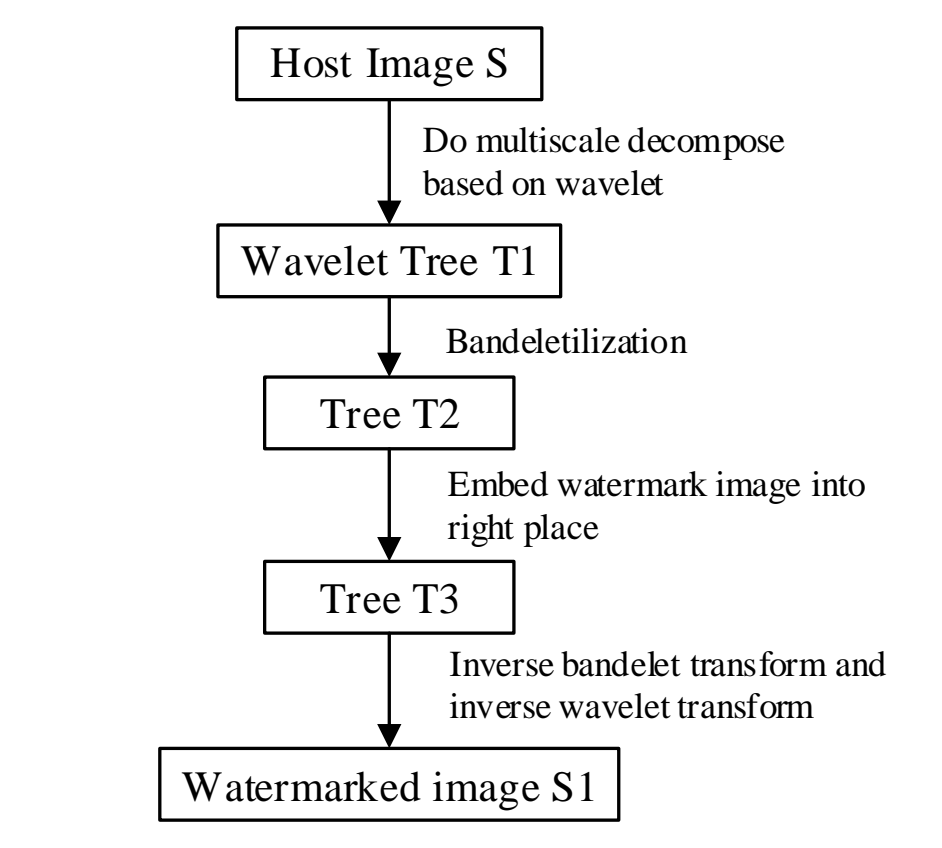

Figure 1. Framework of Watermark Embedding

Step 1) Input host image $S$ and compute its visual sensitivity threshold.

step 2. Take 2D discrete wavelet tree transform of $\mathrm{S}$, and then the host image is transformed to wavelet tree $\mathrm{T} 1$.

Step 3. Convert tree T1 to tree T2 by bandeletization.

Step 4. Input watermark image W, search every image blocks of tree $\mathrm{T} 2$, and select these whose length is not shorter than the length of the watermark image, and then embed watermark image into these image blocks. This tree is represented by T3. In addition, the position where the watermarks are embedded should be stored. The string storing these information is named key string here.

Step 5. Do inverse bandelet transform and inverse discrete wavelet transform of T3, and then watermarked image $\mathrm{S} 1$ come out. 


\subsection{The Extraction Algorithm}

In plain terms, the process of watermark extraction is an inverse process of watermark embedding. These steps are shown in Figure 2.

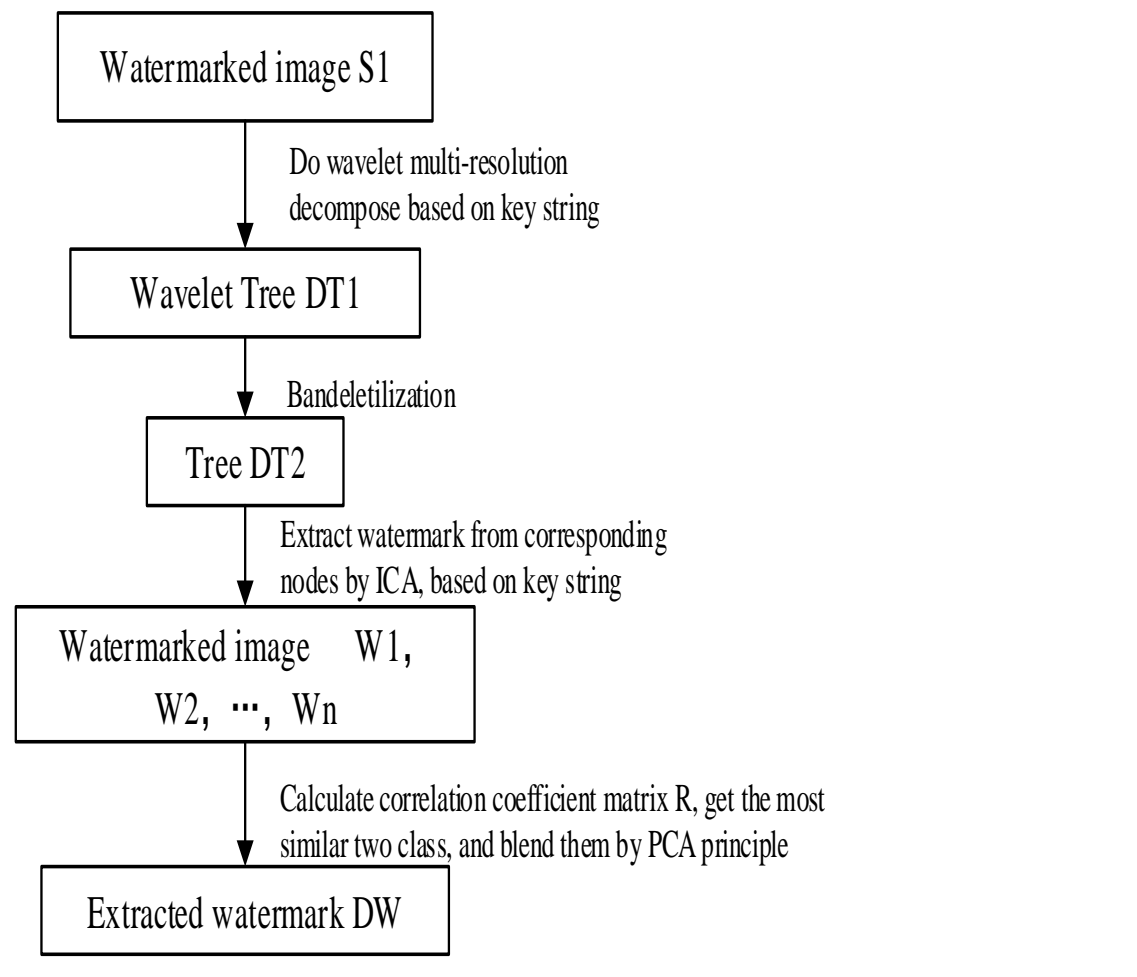

Figure 2. Franeeyvork of Watermark Extraction

Step1. Input watermarked image S1 and Rey string, then calculate size of node size.

Step2. Do wavelet tree decompose to S1, and then wavelet tree DT1 is obtained.

Step3. Convert tree DT1 to tree DT2 by bandeletization.

Step4. Extract watermark image from tree DT2 with independent component analysis.

Independent component analysis (ICA), a method for finding underlying components from multidimensional statistical data, looks for components that are statistically independent [15]. Indeed, ICA is an estimate based on the statistic of information. That is, the independent information are used to estimate the mixed matrix, and then, the sources are separated. This theory can be expressed by (5):

$$
\begin{gathered}
\mathrm{X}=\mathrm{AS} \\
\mathrm{X}=\left\{x_{1}, x_{2}, \cdots, x_{m}\right\} \\
\mathrm{S}=\left\{s_{1}, s_{2}, \cdots, s_{n}\right\}
\end{gathered}
$$

$\mathrm{X}$ ingukidimensional statistical data and $\mathrm{S}$ is statistically independent data. A is mixing natrix.I mixing matrix $A$ is already known, the inverse matrix of mixing matrix is easy to ge. That is, separating matrix, $\mathrm{W}=\mathrm{A}-1$ can be easily got. Y, estimated value of independent data $\mathrm{S}$, can be computed by (8):

$$
Y=W X
$$

In order to reduce the amount of computation and promote efficiency of extracting watermark, an ICA method based on mutual information and negentropy is introduced. This method avoids the complexities of matrix inversion, which exists in some other ICA method.

Step 5. Compute the correlation matrix of these watermarks, $\mathrm{R}$, and find the most familiar two watermark and blend them to attain the final extracted watermark.

Image fusion, an application in digital image processing of data fusion technique, is an important branch of data fusion, which aims at gaining an accurate, comprehensive and 
reliable description of the same image to improve accuracy rating of detecting or recognizing target, through extracting and integrating multiple images information [16]. Because extracted watermark may be partially broken after cover image is attacked, in order to improve the quality of extracted watermark, we adopt image fusion method to fuse those extracted broken watermark images to get the correct watermark image. This step is very important to achieve the most familiar between the extracted watermark image and the original watermark image. Principal component analysis (PCA) is a commonly used image fusion method, and already applied in image compress, feature selection and so on [17]. It is an optimal orthogonal exchange on the aspect of least mean square, which is used to achieve watermark image fusion in this paper. Every extracted watermark image is treated as a random variable $x_{n}$. Then, the extracted watermark images construct a random vector $\mathrm{X}$.

$$
X=\left(x_{1}, x_{2}, \cdots, x_{n}\right)^{T}
$$

Mean value of $\mathrm{X}$ is $m_{x}, m_{x}=E\{X\}$. Covariance matrix of $\mathrm{X}$ is $C_{x}$.

$$
C_{x}=\mathrm{E}\left\{\left(X-\mu_{x}\right)\left(X-\mu_{x}\right)^{T}\right\}
$$

The feature value $l_{i}$ and the feature vector $e_{i}$ should satisfy this relation hip:

$$
C_{x} e_{i}=\lambda_{i} e_{i} \quad i=1, \cdots, n
$$

After feature values and feature vectors of square matrix are computed, feature vectors are ranked by the feature value in descending. Then the transform matrix, A, is applied on transforming X:

$$
\mathrm{Y}=\mathrm{A}\left(\mathrm{X}-\mu_{\mathrm{N}}\right)
$$

The first principle component of $\mathrm{Y}$ is the fused image.

This method possess a high efficiency on-mage firsion, but it treat every pixel same, which will neglect image blocks with 10 wenergy In the process of image fusion, because most watermark images have been attacked and some watermark information's energy of watermarked image are already low, if these watermark are treated equally without discrimination, the result may be error 118. So, this paper proposed a method: first, compute the correlation matrix between damaged watermarks, then rank the extracted watermark according to correlation coefficients value, and at last fuse the front watermark data to achieve a better result.

\section{Experimental Results and Analysis}

\subsection{Experiment Setting}

In our experimentalion, Matlab8.3 is used as the emulator and the standard image Lena with size $512 \times 512 \times 8$ bit is chosen as the host image and the watermark image is a $40 \times 40$ binary image

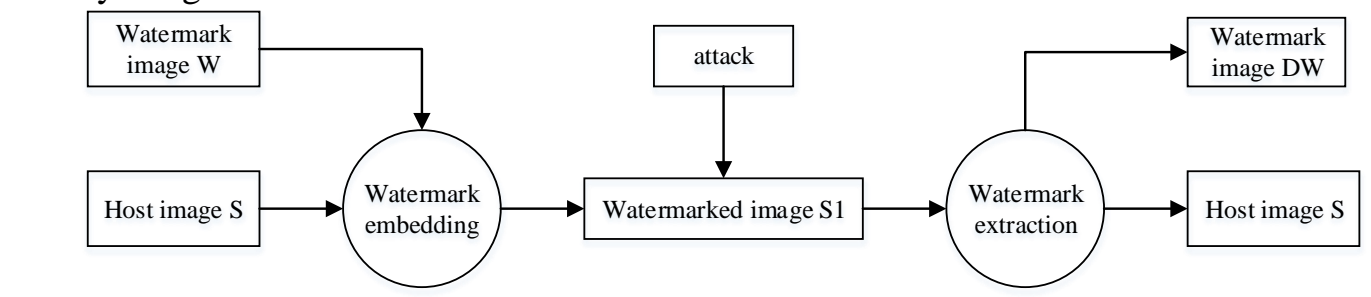

Figure 3. Framework of Experimentation

Figure 6, as shown below, is the watermarked image, and its PSNR value is $38.0177 \mathrm{~dB}$, which demonstrate that this watermark method perform well on the aspect of imperceptibility. 


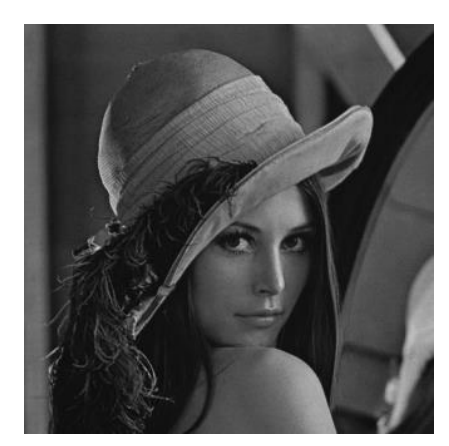

Figure 4. Host Image

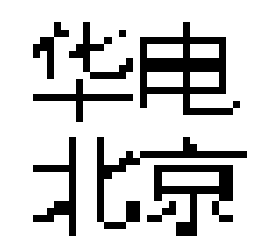

Figure 5. Watermark Image

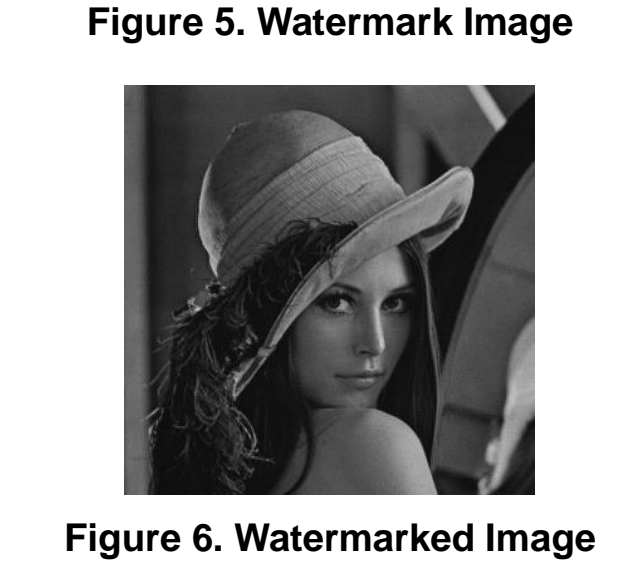

Under the situation of no attack. the result of detecting watermark from Figure6 is the same as Figure5, That is, the extracted watermark image is same as the original watermark image exactly.

\subsection{Robustness Test and Analysis}

Gaussian noise of multiplicative noise or other attack to Figure 3. Figure 7 show result that Figure 3 after these kinds of attack.

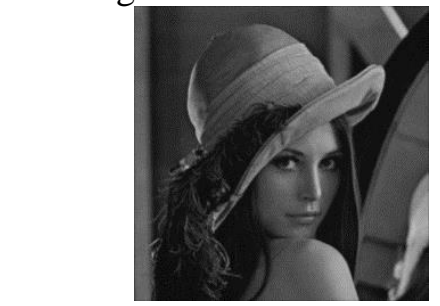

a. Gausssian Low Pass

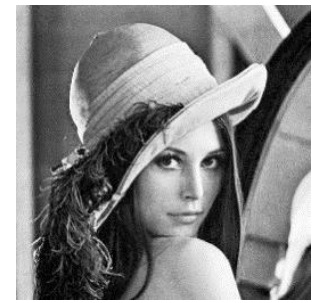

b. Histogram Equalization

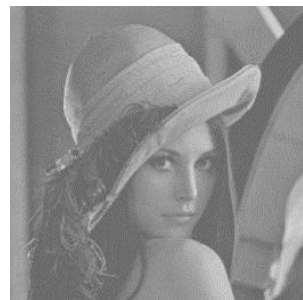

c. Enhancement 30\% 


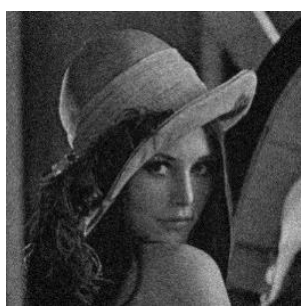

d. $1 \%$ Gaussian Noise

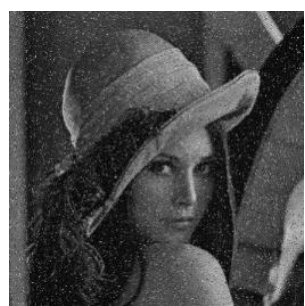

e. $6 \%$ Salt and Pepper Noise

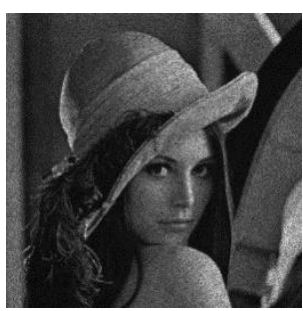

f. $8 \%$ Multiplicative Noise

Figure 7. Watermarked Image after Attack

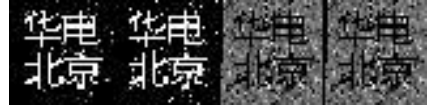

(a) Detected Watermark

\section{华电 \\ 北京}

Figure 8. Watermark Extract and Detect after Gaussian Low Pass

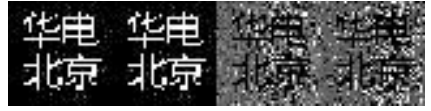

(a) Detected Watermark

(b) Fused Watermark

(b) Fused Watermark

Figure 9. Watermark Extract and Detect after Histogram Equalization

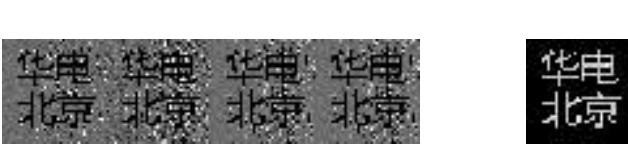

(a) Detected Watermark (b).Fused Watermark

Figure 10. Watermark Extract andDetect after Enhancement 30\%

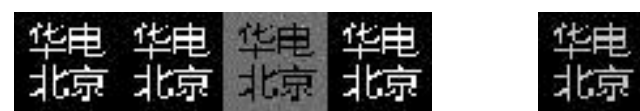
(a) Detected Wate mark
(b) Fused Watermark

Figure 11. Watermark Extract and Detect after 1\% Gaussian Noise

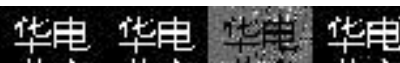

北京 北京 北票 北京 北京
(a) Detected Watermark
(b) Fused Watermark

Figure 12. Watermark Extract and Detect after 6\% Salt and Pepper Noise

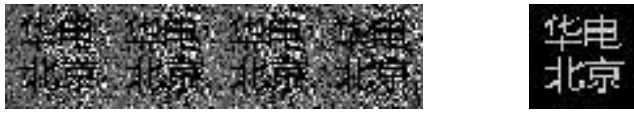
(a) Detected Watermark
(b) Fused Watermark

Figure 13. Watermark Extract and Detect after $8 \%$ Multiplicative Noise

Table1 shows the robustness of the algorithm proposed by this paper, through comparing with robustness of algorithm proposed by paper [19].

Table 1. Correlation Coefficient (CORR) Result after Attack 


\begin{tabular}{lll}
\hline Attack & CORR(this paper) & CORR (paper [19]) \\
\hline Gaussian low pass & 0.9991 & 0.9912 \\
Histogram Equalization & 0.9975 & 0.9975 \\
enhancement 30\% & 0.9930 & 0.9937 \\
1\% gaussian noise & 0.9996 & 0.9970 \\
6\% salt and pepper noise & 0.9990 & 0.9938 \\
8\% multiplicative noise & 0.9907 & 0.9856 \\
\hline
\end{tabular}

Table1. shows that, compared to robust watermarking algorithm proposed by [19], the scheme proposed by this paper show more robust in most cases. It also demonstrates that information hidden in bandelet transform domain can resist most common attack more effectively than wavelet transform domain. Meanwhile, the image fusion technology also help improve the robustness of the proposed watermark scheme.

\subsection{Imperceptibility Analysis}

As Figure 6 showing up, the watermarked image seems the same as the on ginal one. Due to the tree shape structure of the bandelet transform, this scheme keeps well watermark imperceptible. So these results have proved that the proposed scheme has achieved a good balance between imperceptibility and robustness.

\section{Conclusion}

In this paper, we have proposed a robust drgital watermarking scheme based on second generation of bandelet transform. Because the non-tero wavelet coefficients have been processed through bandeletization, mos information has been concentrated on some main regions. Therefore, this scheme possessed a strong robustness against the noise attacks. Experimental results also demonstrate that the watermarking algorithm based on bandelet has a good performance both in imperceptibility and robustness. Compared to the common watermarking algorithms the proposed scheme can achieve a better balance between imperceptibility and robusthess.

\section{References}

[1] H. Tao, L. Chongmin, J, Monamad Zain and A. N. Abdalla, "Robust Image Watermarking Theories and Techniques: A Review" I Lournal of Applied Research and Technology, vol. 12, no. 1, (2014), pp. 122-138.

[2] J. S. Li, “A novel blind color images watermarking based on SVD”, J. Optik, vol. 125, no. 12, (2014), pp. 2868-2874.

[3] B. L. Gunjal and S N. Mali, "Comparative performance analysis of digital image watermarking scheme in DWT and DWTFWHT-SVD domains", Proceedings of the India Conference (INDICON), 2014 Annual IEEE, Pune. India, (2014), pp. 1-6.

[4] L. Yuancheng and W. Xiaolei, "A Novel Multiple Watermarking Algorithm Based on Bandelet Transform”, Procedings of the 2009 WRI Global Congress on Intelligent Systems, Xiamen, China, (2009), pp. 238-242.

15] T. T. Wang, J. Q. Ni and J. W. Huang, “An Informed Watermarking Scheme Using Hidden Markov Model in the Wavelet Domain", J. IEEE Transactions on Information Forensics and Security, vol. 7, no. 3, (2012), pp. 853-867.

[6] B. Ning, S. Qiyu, H. Daren, Y. Zhihua and H. Jiwu, "Robust Image Watermarking Based on Multiband Wavelets and Empirical Mode Decomposition", Journal Image Processing, IEEE Transactions on, vol. 16, no. 8, (2007), pp. 1956-1966.

[7] D. Kundur and D. Hatzinakos, "Digital watermarking for telltale tamper proofing and authentication", Journal Proceedings of the IEEE, vol. 87, no. 7, (1999), pp. 1167-1180.

[8] M. Zareian and H. R. Tohidypour, "Robust quantisation index modulation-based approach for image watermarking”, J. Image Processing, IET, vol. 7, no. 5, (2013), pp. 432-441.

[9] V. Senthil and R. Bhaskaran, "Digital image watermarking using edge detection and wavelets with robustness analysis against JPEG compression attacks", Proceedings of the 2008 International Conference on Innovations in Information Technology, Al Ain, United Arab Emirates, (2008), pp. 583-587.

[10] X. Liang, Z. H. Wei and H. Z. Wu, "In Interactive Technologies and Sociotechnical Systems", Edited H. Zha, Z. Pan, H. Thwaites, A. C. Addison and M. Forte, Springer Berlin Heidelberg, Xi'an, vol. 4270, 
(2006), pp. 119-127.

[11] E. Le Pennec and S. Mallat, "Image compression with geometrical wavelets", Proceedings of the 2000 International Conference on Image Processing, Vancouver, British Columbia, Canada, (2000), pp. 661-664.

[12] E. Le Pennec and S. Mallat, "Sparse geometric image representations with bandelets", Journal IEEE Transactions on Image Processing, vol. 14, no. 4, (2005), pp. 423-438.

[13] M. J. Tsai and C. W. Lin, "Wavelet based multipurpose color image watermarking by using dual watermarks with Human Vision System models", J. Ieice Transactions on Fundamentals of Electronics Communications and Computer Sciences, vol. 91, no. 6, (2008), pp. 1426-1437.

[14] J. F. C. Wanderley and M. H. Fisher, "Multiscale color invariants based on the human visual system", Journal Image Processing, IEEE Transactions on, vol. 10, no. 11, (2001), pp. 1630-1638.

[15] M. D. Plumbley, "Algorithms for nonnegative independent component analysis", Journal Neural Networks, IEEE Transactions on, vol. 14, no. 3, (2003), pp. 534-543.

[16] I. Kosesoy, A. Tepecik, M. Cetin and A. Mesut, "A comparative analysis of image fusion methods", Proceedings of the 20th Signal Processing and Communications Applications Conference, Fethiye, Mugla, Turkey, (2012), pp. 1-4.

[17] K. Hui, L. Xuchun, W. Lei, T. E. Khwang, W. J. Gang and R. Venkateswarlu, "Generalized 2D principal component analysis", Proceedings of the 2015 IEEE International Joint Conference on Neural Networks, Hilton Montreal Bonaventure Montreal, QC, Canada, (2005), pp. 108-113.

[18] C. T. Shou, T. T. Hsin, C. Y. Tzu, L. C. Chiang, C. R. Chang, L. S. Yow and C. H Yi, "A combined K-means and hierarchical clustering method for improving the clustering efficiency of microarray", Proceedings of the 2005 International Symposium on Intelligent Signal Processing and Communication Systems, Hong Kong, China, (2005), pp. 405-408.

[19] S. Wei, S. X. Ming and Y. H. Fu, "Robust Digital Watermarking Algorithm Based on Data Fusion and ICA", J. Computer Engineering, vol. 34, no. 10, (2008), pp. 150-152+157.

\section{Authors}

Yuancheng Li, received the Ph.D. degrea from Uniersity of Science and Technology of China, Hefei, China, in 2003. From 2004to 2005 he was a postdoctoral research fellow in the Digital Media Lab, Beihang University, Beijing China. Since 2005, he has been with the North China Electric Power University, where he is a professor and the Dean of the Institute of Smart Grid and Information Seeunity. From 2009 to 2010, he was a postdoctoral research fellow in the Cyer Security Lab Pennsylvania State University, Pennsylvania, USA. His current research interests include Cyber Security and information security.

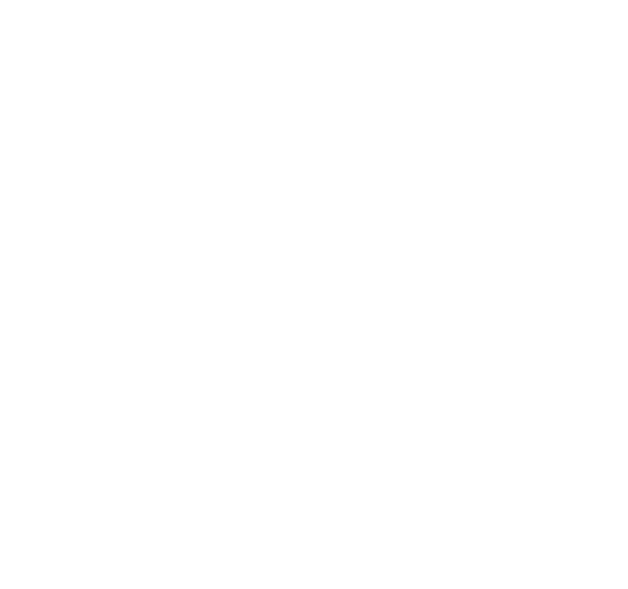




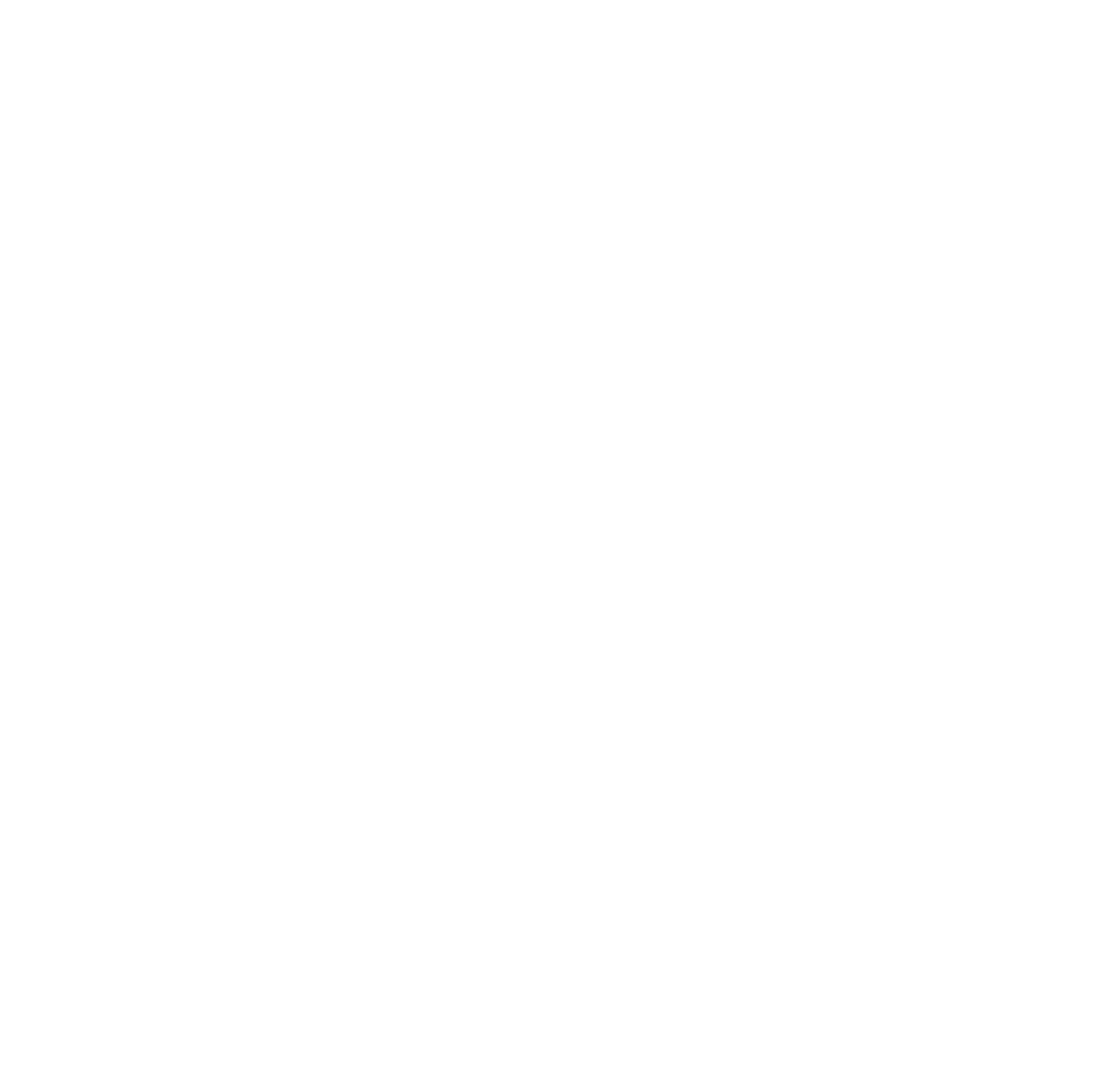

